\title{
The Unfolding Effects of Urea on the Myoglobin-Imidazole Complex Studied by Cyclic Voltammetry and Visible Absorption Spectroscopy
}

\author{
Percy L. TORKORNOO and Fred M. HAWKRIDGE* \\ Received June 6, 1994 ; Accepted January 20, 1995
}

\begin{abstract}
The unfolding effects of urea on the myoglobin-imidazole complex have been studied using cyclic voltammetry and visible absorption spectroscopy. Since about $70 \%$ of the protein of myoglobin is in an alpha helix, changes in both cathodic and anodic peak potentials, peak currents, diffusion coefficient, and UV-VIS spectra are expected when myoglobin unfolds due to urea. Interestingly, with increasing concentration of urea the visible Soret band of myoglobin decreases and both anodic and cathodic currents decrease. Using these criteria the effective concentration of myoglobin decreased by $12.5 \%$ in $3 \mathrm{M}, 21.3 \%$ in $6 \mathrm{M}$, and $30.8 \%$ in $8 \mathrm{M}$ aqueous urea solution.
\end{abstract}

\section{INTRODUCTION}

Myoglobin, the oxygen storage protein found in mammalian muscles, has a single polypeptide chain of 153 amino acids. It is a compact molecule with the overall dimensions of $4.5 \times 3.5 \times 2.5 \mathrm{~nm}{ }^{1,2)}$. About $70 \%$ of the molecular chain of myoglobin is folded into eight alpha helices. Polar groups cover the molecule's surface while the interior is almost entirely composed of nonpolar groups which lead to the highly globular structure of myoglobin 3 ).

The prosthetic group of myoglobin, as well as of hemoglobin, is the very stable iron-porphyrin coordination compound, or heme. The heme is held in a pocket in the protein structure only by coordination of the heme iron to an imidazole nitrogen on a histidine residue. The physiologically active oxidation state of the heme iron is +2 . The sixth coordination site in myoglobin in this oxidation state is either vacant or occupied by oxygen. In the +3 heme iron oxidation state this sixth coordination site is occupied by water. In both myoglobin ${ }^{3)}$ and

Department of Chemistry,

Virginia Commonwealth University,

(Box 842006, Richmond, VA 23284 U.S.A.)

Key Words: Myoglobin, visible absorption spectroscopy, cyclic voltammetry. hemoglobin ${ }^{4)}$, the binding of the heme to a ligand produces conformational changes in the protein structure. The interaction between the heme prosthetic group and the protein structure results in a stable complex which binds reversibly to oxygen without oxidation of the iron for both molecules ${ }^{3,4)}$.

There have been extensive studies to probe the conformational changes of myoglobin that are associated with both its electron transfer and its ligand binding reactions. Transient kinetic methods have been used to elucidate the mechanisms of ligand binding and dissociation including circular dichroism and $\mathrm{NMR}^{5)}$, flash photolysis ${ }^{6,7)}$, stopped-flow spectroscopy ${ }^{8,9)}$, fluorescence lifetimes ${ }^{10)}$, molecular dynamics simulations ${ }^{11)}$ and cyclic voltammetry ${ }^{12)}$. However, the mechanism by which solution resident oxygen actually arrives at the heme iron for binding in myoglobin remains to be elucidated as does the reverse case for complete dissociation.

The structure of native myoglobin is more resistant to unfolding than is the form with the heme iron removed, apomyoglobin. The presence of the heme unit in myoglobin stabilizes the protein structure. At neutral pH, apomyoglobin is therefore less stable with an alpha helical content reduced to about $55 \%$ from its $70 \%$ value for the native structure as measured by circular dichroism ${ }^{5}$ ) 
However, the compactness of native myoglobin and apomyoglobin is indistinguishable as judged by intrinsic viscosity measurements ${ }^{13}$ ). These results indicate that the structure of apomyoglobin is not substantially unfolded compared with the native myoglobin structure ${ }^{7)}$.

Urea is widely used as a powerful denaturing agent for probing the robustness of protein structure in aqueous solution. Globular proteins, including myoglobin, usually undergo loss of tertiary structural features in the presence of urea ${ }^{1)}$ and these transitions are usually completed at urea concentrations between $6 \mathrm{M}$ and $8 \mathrm{M}$, except for unusually stable proteins ${ }^{14)}$.

In the work presented here, cyclic voltammetry and visible absorption spectroscopy are used to study the changes that occur when solutions of the myoglobin-imidazole complex are subjected to increasing concentrations of urea. The complex between oxidized myoglobin and imidazole has been chosen for this study because reduction of the heme iron leads to dissociation of the imidazole ligand. This affords a means of studying the effects of urea induced changes in the structure of myoglobin upon the properties of the ligand dissociation reaction using simple cyclic voltammetric measurements. Changes in peak currents and potentials for both reductive and oxidative electron transfer reactions, diffusion coefficients, and visible absorption spectra are described.

The ultimate aim of this work is to better understand the mechanism that describes the dissociation of oxygen from its heme iron coordination site and then its release from the fabric of the myoglobin molecule so that it is available for reaction in the terminal step of mammalian respiration.

\section{EXPERIMENTAL}

Horse skeletal myoglobin was obtained from Sigma Chemical Company. Myoglobin solutions were prepared by dissolving lyophilized solid in tris(hydroxymethyl)aminomethane/cacodylic acid buffer, $\mathrm{pH} 7.0$ and then filtering these solutions through a 30,000 molecular weight filter (YM30. Amicon Div., Grace and Co.) just before each experiment. This buffer (ionic strength $0.1 \mathrm{M}$ ) was chosen to minimize ion binding of buffer components to the protein such as occurs between phosphate and myoglobin ${ }^{15,16)}$. The tris(hydroxymethyl)aminomethane (Trizma Base, Reagent grade, Sigma) was used as received whereas the cacodylic acid (hydroxydimethylarsine oxide, $98 \%$ pure) was recrystallized twice from 2-propanol. Nitrogen (grade 4.5 from Airco, Inc.) was passed over hot copper turnings, $550^{\circ} \mathrm{C}$, in a Sargent-Welch furnace to remove oxygen, bubbled through water to minimize sample evaporation and then bubbled through the sample solution to remove oxygen. Tindoped indium oxide deposited on glass (Donnelly Corp.) was used as the working electrode in each experiment. Each electrode was cleaned ${ }^{17)}$ by sonicating for 5-min in Alconox detergent solution, in ethanol and twice in distilled water. The reference electrode was $\mathrm{Ag} / \mathrm{AgCl}(1.0 \mathrm{M} \mathrm{KCl})$ and a platinum wire was used as the auxiliary electrode.

All water used was purified with a Milli RO4/Milli Q system (Milli Corp.) which on delivery exhibited a resistivity of $18 \mathrm{M} \Omega \cdot \mathrm{cm}$. The myoglobin concentration was determined spectrophotometrically from the absorbance of solutions of the oxidized and reduced myoglobin at 434 nm using a Hewlett Packard HP8452 diode array spectrophotometer interfaced to an IBM PC computer. The difference molar absorptivity ${ }^{18)}$ at this wavelength is $96,700 \mathrm{M}^{-1} \mathrm{~cm}^{-1}$. After obtaining the spectrum for the oxidized species, the ferrimyoglobin was then reduced with sodium dithionite and the concentration of myoglobin was calculated from these spectra. The concentration of myoglobin was made to be ca. $80 \mu \mathrm{M}$ and a weighed amount of solid imidazole was added to each solution to make a myoglobin to imidazole molar concentration ratio of 1 to 1000 . This insured that essentially all of the myoglobin was present as the myoglobin-imidazole complex. A Lucite cell ${ }^{19)}$ of conventional design was used for all the cyclic voltammetry (CV) experiments. The solution volume was approximately $5 \mathrm{~mL}$ and provision was made for purging nitrogen through the solution to remove oxygen as mentioned above.

Cyclic voltammograms were acquired using a computer data acquisition system that has been described earlier ${ }^{20)}$. Cyclic voltammograms were initially obtained on oxygen free buffer solutions that contained no myoglobin. The cyclic voltammograms 
of myoglobin-imidazole complex solutions were then acquired. Solid urea was then weighed and added to the $5.0 \mathrm{~mL}$ stirred sample solution so that the urea concentration was $3 \mathrm{M}$. Cyclic voltammograms of this solution in the presence of $3.0 \mathrm{M}$ urea were then obtained. Similarly, cyclic voltammograms were obtained on the myoglobin-imidazole complex solutions in the presence of $6 \mathrm{M}$ and $8 \mathrm{M}$ urea. The background cyclic voltammograms obtained on solutions containing only buffer were digitally subtracted from the cyclic voltammograms obtained on solutions of the myoglobin-it.nidazole complex in the presence of varying amounts of urea. The visible absorption spectra of each of these myoglobin-imidazole complex solutions were taken using the instrument described above. The initial myoglobin-imidazole complex solution and the solution containing $8 \mathrm{M}$ urea were each reduced with sodium dithionite and visible absorption spectra were obtained as before.

\section{RESULTS AND DISCUSSION}

The overall electrode reaction mechanism that describes the results presented in this paper can be given by the following equations;

$$
\begin{aligned}
& \mathrm{Mb}(\mathrm{III}) \mathrm{H}_{2} \mathrm{O}+\mathrm{Im} \rightleftarrows \mathrm{Mb}(\mathrm{III}) \mathrm{Im} \\
& \mathrm{Mb}(\mathrm{III}) \mathrm{Im}+\mathrm{e}^{-} \rightleftarrows \mathrm{Mb}(\mathrm{II}) \mathrm{Im} \\
& \mathrm{Mb}(\mathrm{II}) \mathrm{Im} \rightleftarrows \mathrm{Mb}(\mathrm{II})+\mathrm{Im}
\end{aligned}
$$

where $\mathrm{Mb}$ (III) $\mathrm{H}_{2} \mathrm{O}$ is the oxidized, metmyoglobin state, $\mathrm{Im}$ is imidazole, $\mathrm{Mb}$ (III) Im is the myoglobinimidazole complex and $\mathrm{Mb}(\mathrm{II})$ is reduced myoglobin. As described earlier in this work, a large imidazole to myoglobin molar ratio is used so that the reaction shown in Eq. (1) is driven far to the right and it is the myoglobin-imidazole complex that undergoes reduction at the electrode surface. Upon reduction the reduced myoglobin-imidazole complex dissociates and that is the reaction of particular interest to this study. This electrochemically initiated ligand dissociation reaction is analogous to the physiological case in which oxygen dissociates when required by respiration.

Figure 1 shows the background subtracted cyclic voltammograms of a solution containing 78

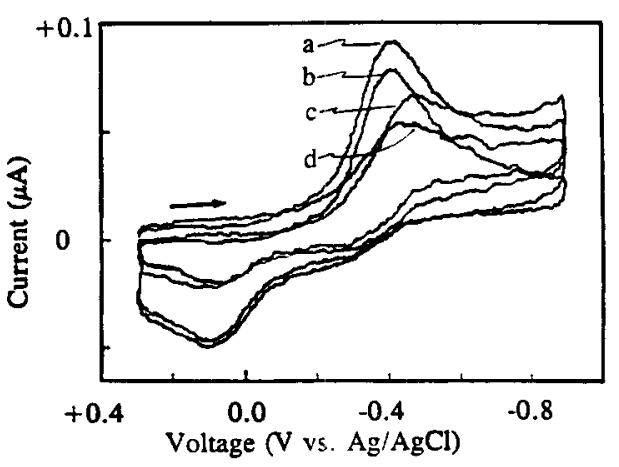

Fig. 1. Background subtracted cyclic voltammograms of $78 \mu \mathrm{M}$ myoglobin-imidazole complex in the presence of the following concentrations of urea a) $0 \mathrm{M}$, b) $3 \mathrm{M}$, c) $6 \mathrm{M}$ and d) $8 \mathrm{M}$.

$\mu \mathrm{M}$ myoglobin-imidazole complex in the presence of $0 \mathrm{M}, 3 \mathrm{M}, 6 \mathrm{M}$ and $8 \mathrm{M}$ urea concentration. At the high myoglobin to imidazole molar ratio used in these experiments the cyclic voltammetry is essentially due to only the reaction given by Eq. 2 . With the increasing concentration of urea, there is a decrease in both cathodic and anodic peak currents and this will be discussed further below. An intriguing aspect of the cyclic voltammetric results is the constancy of the anodic peak potential as a function of urea concentration. This suggests that as the protein is being denatured by increasing urea concentration the oxidation reaction mechanism is not changing although the concentration of the native myoglobin-imidazole complex is decreasing.

The decrease in the visible Soret band of myoglobin-imidazole complex with increasing concentration of urea is shown in Figure 2 for both the oxidized and reduced forms. Upon reduction the myoglobin-imidazole complex dissociates according to Eq. 3. It is interesting that there is a substantial difference between the absorbance of the myoglobinimidazole complex when the urea concentration is changed from $6.0 \mathrm{M}$ to $8.0 \mathrm{M}$ while the analogous electrochemical response is not affected so greatly. As shown in Figure 3 the cathodic peak currents decrease in a nearly linear fashion upon increasing the concentration of urea from $0 \mathbf{M}$ to $8 \mathrm{M}$. No significant change was observed in the peak cathodic or anodic potentials except for the negative shift in the cathodic peak potential at a urea concentration of $6 \mathrm{M}$. This result is reproducible but the origin of 


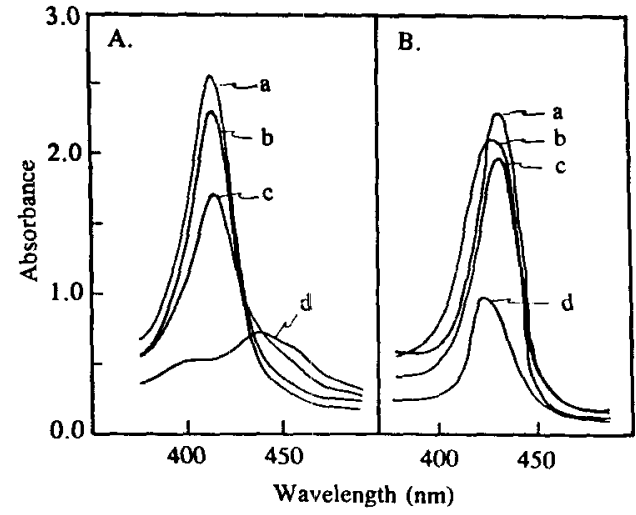

Fig. 2. Absorption spectra for $78 \mu \mathrm{M}$ myoglobinimidazole complex solutions in the A) oxidized and $B$ ) reduced states in the presence of the following concentrations of urea a) $0 \mathrm{M}$, b) $3 \mathrm{M}$, c) $6 \mathrm{M}$ and d) $8 \mathrm{M}$.

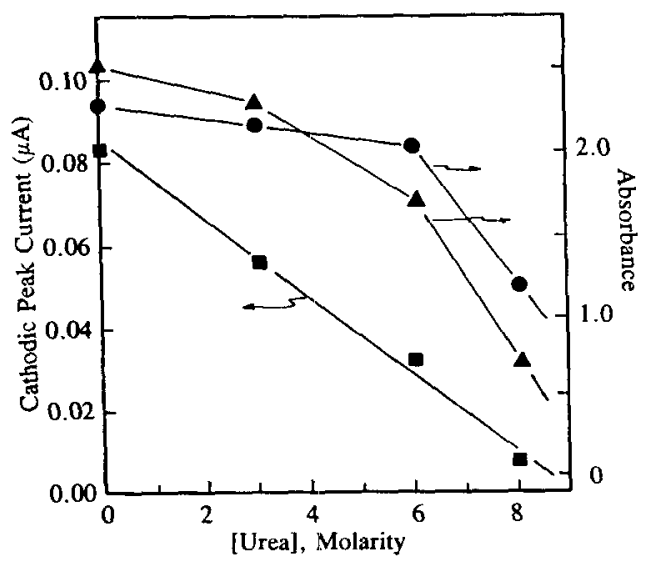

Fig. 3. The dependence of cathodic peak current and Soret absorbance maximum for $78 \mu \mathrm{M}$ myoglobin-imidazole complex where $\boldsymbol{\theta}$ ) is peak cathodic current, 4 ) is absorbance of oxidized solution and $\bullet$ ) is absorbance of dithionite reduced solution.

this shift is not presently understood. There clearly is a decrease in the anodic peak currents for the two higher urea concentrations studied. The reduction in both the visible Soret band and the peak cathodic and anodic currents is evidence of denaturing of the myoglobin-imidazole complex ${ }^{21)}$. The magnitude of the peak cathodic current can be described by the
Randles-Sevcik equation ${ }^{22}$ ) and the data shown in Figure 1 were analyzed using this relationship. Using a diffusion coefficient of $1.1 \times 10^{-6} \mathrm{~cm}^{2} / \mathrm{s}^{20}$, the effective concentration of myoglobin was found to be reduced by $13 \%$ in $3 \mathrm{M}, 21 \%$ in $6 \mathrm{M}$, and 31 $\%$ in $8 \mathrm{M}$ urea solution.

As the myoglobin molecules unfold the population of the denatured molecules increases and we expect an increase in entropy as the polypeptide unfolds leading to the complete exposure of the heme. During the process of unfolding the imidazole-heme complex fully dissociates from the protein of the myoglobin molecule. It is expected that as heme gets exposed the peak currents and the Soret band would increase due to increased accessibility to the electrode surface. However, the peak currents decrease with increasing urea concentration. One possible explanation for this observation may be that with the increasing concentration of urea the solubility of myoglobin decreases $^{21)}$. Although no precipitation was evident visually, this could possibly explain these results. Another possible explanation is that in its denatured state, the redox potential of the free heme is shifted negative of the potential window accessible in this work thereby decreasing the observed peak currents. .

The results presented here suggest that cyclic voltammetry can be a very sensitive probe for monitoring the process of unfolding of myoglobin as a function of urea concentration. It would be valuable to determine if this effect could be reliably used to characterize the oxygen binding and dissociation reactions of myoglobin under similar conditions. This will require optimization of the heterogeneous electron transfer reaction of metmyoglobin 23 ) in the presence and absence of oxygen.

We gratefully acknowledge the National Science Foundation (grant C.HE 9111786) and the Department of Chemistry, Virginia Commonwealth University for providing the financial support for this work.

\section{REFERENCES}

1) L. Stryer, Biochemistry, W. H. Freeman and Co., New York, p. 145 (1988). 
2) L. J. Kagen, Myoglobin, Columbia Univ. Press, New York, p. 13 (1973).

3) R. E. Dickerson and I Geis, Hemoglobin: Structure, Function, Evolution and Pathology, The Benjamin/Cummings Pub. Co., Menlo Park, CA, pp. 26-33 (1983).

4) ibid, pp. 34-60.

5) F. M. Hughson, P.E. Wright and R. L. Baldwin, Science, 249, 1544 (1990).

6) S. Balasubramanian, D. G. Lambright, M. C. Marden and S. G. Boxer, Biochemistry., 32, 2202 (1993).

7) F. Sato, Y. Shiro, Y. Sakaguchi, T. lizuka and H. Hayashi, J. Biol. Chem., 265, 18823 (1990).

8) K. Tsukahara, J. Am. Chem. Soc., 111, 2040 (1989).

9) A. Bellelli, G. Antonini, M. Brunori, B. A. Springer and S. Sligar, J. Biol. Chem., 265, 18898 (1990).

10) E. Bismuto, E. Gratton and G. Irace, Biochemisty, 27, 2132 (1988)

11) K. Kuczera, J-C. Lambry, J-1. Martin and M. Karplus, Proc. Nat. Acad. Sci. USA, 90, 5805 (1993).

12) B. C. King and F. M. Hawkridge, Talanta, 36, 331 (1989).

13) Yu. V. Griko, P. L. Privalov, S. Yu. Venyaminov and V. P. Kutyshenko, J. Mol. Biol., 202, 127 (1988).
14) C. N. Pace, CRC Critical Review Biochem., 3, 1 (1975).

15) E. F. Bowden, F. M. Hawkridge and H. N. Blount, Electrochemical and Spectrochemical Studies of Biological Redox Components (Ed. K. M Kadish) American Chemical Society, Washington, D.C., p. 159 (1982).

16) J.M. Gillespie, K. D. Hapner, C. R. Hartzell and F. R. Gurd, J. Mol. Biol., 21, 399 (1966).

17) N. R. Armstrong, A. W. C. Lin, M. Fujihira and T. Kuwana, Anal. Chem., 48, 741 (1976).

18) E. F. Bowden, F. M. Hawkridge and H. N. Blount, J. Electroanal. Chem., 116, 447 (1980).

19) E. F. Bowden, Ph.D. Dissertation, Virginia Commonwealth University (1982).

20) B. C. King, F. M. Hawkridge and B. M. Hoffman, J. Am. Chem. Soc., 114, 10603 (1992).

21) L. R. De Young, K. A Dill and A. L. Fink, Biochemistry, 32, 3877 (1993).

22) R. N. Adams, Electrochemistry at Solid Electrodes, Marcel Dekker Inc., New York, p. 124 (1969).

23) I. Taniguchi, K. Watanabe, M. Tominaga and F. M. Hawkridge, J. Electroanal. Chem., 333, 331 (1992). 